\title{
Efeito da atividade física em ambiente enriquecido sobre o músculo esquelético de camundongos com caquexia associado ao melanoma cutâneo
}

\author{
Effect of physical activity in an enriched \\ environment on the skeletal muscle of mice with \\ cachexia associated with cutaneous melanoma
}

\author{
Karen Layane dos Santos ${ }^{1}$ \\ Tayrine Resende de Oliveira ${ }^{2}$ (C) \\ João Vitor Nunes Lopes ${ }^{3}$ \\ Alex Sander Freitas 4 \\ Magda Mendes Vieira ${ }^{5}$ \\ Berenilde Valéria de Oliveira Sousa ${ }^{6}$ (1) \\ Mariana Rocha Alves ${ }^{7}$ \\ Vinicius Dias Rodrigues ${ }^{8}$ (1)
}

\begin{abstract}
${ }^{1}$ Autora para correspondência. Universidade Estadual de Montes Claros (Montes Claros). Minas Gerais, Brasil. kklayane@yahoo.com.br ${ }^{2}$ Ambulatório de Neurociências do Exercício, Hospital Aroldo Tourinho (Montes Claros). Minas Gerais, Brasil. tayrineoliveirauni@gmail.com ${ }^{3}$ Faculdade Integradas do Norte de Minas (Montes Claros). Minas Gerais, Brasil. joaollopes@outlook.com.br 4-6Universidade Estadual de Montes Claros (Montes Claros). Minas Gerais, Brasil. alexsanderfreitas3@gmail.com, magdamendesvieira@hotmail.com, berenilde.valeria7@gmail.com ${ }^{7}$ Faculdade Verde Norte (Mato Verde). Minas Gerais, Brasil. marianarochaalves13@gmail.com ${ }^{8}$ Universidade Estadual de Montes Claros (Montes Claros). Minas Gerais, Brasil. viniciuslabex@hotmail.com
\end{abstract}

RESUMO | OBJETIVO: O objetivo deste estudo foi verificar os efeitos da atividade física em ambiente enriquecido sobre histomorfometria do músculo quadríceps femoral de camundongos C57BL/6 com caquexia associada ao modelo tumoral singênico de melanoma cutâneo. MÉTODOS: Foram utilizados camundongos fêmeas da linhagem C57BL/6 que foram distribuídos aleatoriamente em dois grupos: controle CRC $(n=11)$, que não realizou nenhum tipo de intervenção, o segundo grupo foi o experimental (CRC-ATF) ( $n=15)$, que realizou atividade física organizada em ambiente enriquecido de $60 \mathrm{~cm}$ de comprimento, $30 \mathrm{~cm}$ de largura e $45 \mathrm{~cm}$ de altura. Ocorreu indução tumoral de células B16-F10 da linhagem de melanoma cutâneo em todos os animais desse estudo. Após dez dias da indução tumoral, todos os animais já apresentavam quadro de caquexia, assim o grupo experimental iniciou a atividade física em ambiente enriquecido com duração de 30 minutos com intervalos de 48 horas. Após 26 dias do início da intervenção, os animais sobreviventes foram eutanasiados e foi realizada a coleta do músculo quadríceps femoral para análise histomorfométrica. RESULTADOS: Analisando a intervenção realizada, percebemos que o grupo CRC-ATF apresentou a área da fibra muscular maior que o grupo CRC, mas esse resultado não mostrou diferença significativa $(p \leq 0,05)$ entre os grupos. CONCLUSÃO: Os resultados mostram que é possível preservar a estrutura muscular, pois os melhores resultados foram encontrados com os animais que participaram da atividade física em ambiente enriquecido.

PALAVRAS-CHAVE: Aptidão física. Tecido muscular esquelético. Roedores. Câncer. Neoplasia.
ABSTRACT | OBJECTIVE: The objective of this study was to verify the effects of physical activity in an enriched environment on histomorphometry of the quadriceps femoris muscle of C57BL / 6 mice with cachexia associated with the cutaneous melanoma tumor model. METHODS: Female mice of the C57BL / 6 strain were used and were randomly assigned to two groups: CRC control $(n=11)$, which did not perform any type of intervention, the second group was the experimental one (CRC-ATF) ( $n=15$ ), who performed organized physical activity in an enriched environment $60 \mathrm{~cm}$ long, $30 \mathrm{~cm}$ wide and $45 \mathrm{~cm}$ high. Tumor induction of B16-F10 cells of the cutaneous melanoma lineage occurred in all animals in this study. After ten days of tumor induction, all animals already presented with cachexia, so the experimental group started physical activity in an enriched environment lasting 30 minutes with intervals of 48 hours. 26 days after the beginning of the intervention, the surviving animals were euthanized and the quadriceps femoris muscle was collected for histomorphometric analysis. RESULTS: Analyzing the intervention performed, we noticed that the CRC-ATF group had a larger muscle fiber area than the CRC group, but this result showed no significant difference $(p \leq 0.05)$ between the groups. CONCLUSION: The results show that it is possible to preserve the muscular structure, as the best results were found with animals that participated in physical activity in an enriched environment.

KEYWORDS: Physical fitness. Skeletal muscle tissue. Rodents. Cancer. Neoplastic. 


\section{Introdução}

O melanoma é uma neoplasia maligna que se origina a partir dos melanócitos, células que produzem melanina ${ }^{1}$. O melanoma representa cerca de $4 \%$ de todos os tumores da pele, e apesar dessa baixa representatividade é considerado o câncer de pele de maior importância médica, devido à taxa de incidência e mortalidade, podendo se mostrar muito agressivo, pois possui alta capacidade de realizar metástases linfática e hematogênica ${ }^{2}$. Um prognóstico negativo do câncer é a manifestação da caquexia, uma síndrome multifatorial que é caracterizada pela perda de tecido muscular esquelético e tecido adiposo ${ }^{3}$.

A caquexia associada ao câncer é uma síndrome complexa, caracterizada por um estado debilitante, onde ocorre a perda involuntária de peso corporal, perda muscular e alterações metabólicas que ocorrem em 50-80\% dos tumores malignos avançados, representando cerca de $20 \%$ das mortes de pacientes com câncer ${ }^{4}$. Esses fatores beneficiam o tecido tumoral na disputa das fontes energéticas com as demais células do corpo ${ }^{5,6}$. Nesse mesmo aspecto, o nível de gordura corporal sofre considerável redução decorrente do aumento da lipólise e oxidação de lipídeos com baixa na quantidade de lipídeos circulantes, supostamente devido ao aumento da utilização de ácidos graxos para o fornecimento de energia e crescimento tumoral ${ }^{7}$.

Como tratamento alternativo na preservação dessas funções, diversos estudos relatam a atividade física a fim de auxiliar na manutenção do peso e das funções neuromusculares e no combate de estados de fadiga e caquexia ${ }^{\circ}$. Pois, de acordo com Al-Majid e McCarthy ${ }^{9}$, a atividade física possui a capacidade de induzir mudanças tanto na via de síntese, quanto na de degradação proteica, ambas as vias estão relacionadas à perda de tecido muscular em indivíduos oncológicos.

A atividade física é capaz de alterar a funcionalidade imunitária de indivíduos saudáveis, possivelmente, devido às alterações nas concentrações de algumas citocinas. Como a caquexia é causada predominantemente, por citocinas produzidas pelo sistema imunitário do hospedeiro ou pelo tumor, a atividade física pode ser um tipo de intervenção a ser utilizada no tratamento do câncer ${ }^{10}$.

Pensando em melhores estratégias experimentais de exercício ou atividade física com camundongos para intervenção do quadro caquético, Aguiar Jr e Pinho ${ }^{11}$, relatam que estudos com modelos de atividade física em ambiente enriquecido têm resultados importantes na saúde em geral dos roedores, mas ainda, não têm modelos experimentais com caquexia associadas ao câncer que mostram tais benefícios, principalmente, em função da estrutura músculo esquelética. Desta forma, o presente estudo teve como objetivo, verificar os efeitos da atividade física em ambiente enriquecido sobre histomorfometria do músculo quadríceps femoral de camundongos C57BL/6 com caquexia associada ao modelo tumoral singênico de melanoma cutâneo.

\section{Material e métodos}

\section{Caracterizações do estudo e cuidados éticos}

O presente estudo foi analítico, prospectivo e de abordagem quantitativa. O trabalho foi submetido à análise por um comitê de ética em experimentação animal e bem-estar (CEEBEA / Unimontes) e teve parecer favorável para execução (131/2017). Foram utilizados 38 camundongos C57BL/6 fêmeas, foram distribuídos aleatoriamente em dois grupos. O primeiro grupo foi o controle (CRC) $(n=11)$, não realizou nenhum tipo de intervenção, o segundo grupo foi o experimental (CRC-ATF) $(n=15)$, realizou atividade física em ambiente enriquecido. Ocorreu indução tumoral de células B16-F10 da linhagem de melanoma cutâneo em todos os animais desse estudo.

Antes, foi realizado um experimento para definição da caracterização da caquexia no modelo singênico de melanoma cutâneo com uso de linhagem de melanoma murino B16-F10, para este ensaio foram utilizados 12 animais $\frac{12}{}$.

\section{Indução tumoral}

Os animais C57BI/6 são indicados em experimentos para implante de tumores, com destaque para o modelo singênico de melanoma cutâneo com uso de linhagem de melanoma murino B16-F10. Primeiramente, o camundongo C57BI/ 6 foi imobilizado e submetido à tricotomia da região dorsal, próxima ao pescoço. Em seguida, foi verificada a ausência de sensibilidade dolorosa reflexa. Após assepsia com álcool iodado, os animais foram inoculados com 5×105 células de melanoma murino B16-F10, ressuspensas 
em $50 \mu \mathrm{L}$, na região subcutânea dorsal, próxima à base do pescoço (flanco). A inoculação dessa quantidade de células viáveis na região subcutânea tem a capacidade de concluir um ciclo mitótico em $24 \mathrm{~h}$ e de desenvolver o tumor no prazo de 3-4 dias ${ }^{12}$.

\section{Formato experimental da atividade física}

Para evitar comprometer os protocolos experimentais, ocorreram duas sessões de 30 minutos de atividade física (AF) em ambiente enriquecido antes da indução tumoral com objetivo de familiarização dos camundongos do grupo experimental.

A AF foi organizada em um ambiente enriquecido de $60 \mathrm{~cm}$ de comprimento, $30 \mathrm{~cm}$ de largura e $45 \mathrm{~cm}$ de altura. Esse ambiente foi combinante com gangorra, rodas, bolas e túneis, todos os animais ficaram juntos durante 30 minutos por sessão. Ocorreram sete sessões com intervalos de 48 horas. Durante as sessões não foram controlados a movimentação, velocidade e deslocamento do animal ${ }^{13}$. Os animais tiveram livre acesso a água filtrada e ração balanceada (PurinaLabina $\left.{ }^{\circledR}\right)$ no local de alojamento no bioterio.

\section{Diagnóstico da caquexia}

A caquexia relacionada à melanoma cutâneo $(\mathrm{MC})$ foi estabelecida assim que os camundongos portadores do modelo $M C$ singênico mostraram uma perda de pelo menos $5-10 \%$ do peso corporal (desconsiderando o peso do tumor) durante a progressão do tumor ${ }^{14}$. Uma equação de regressão linear foi usada para definir a relação entre o volume do tumor e o peso do tumor. As medições foram realizadas em 12 dias após a inoculação de células tumorais em uma amostra de 12 animais, a fim de calcular o peso do tumor durante todo o experimento. A cada dia, um camundongo foi eutanasiados e o volume do tumor $(\mathrm{mm} 3)$ e a massa $(\mathrm{g})$ foram medidos para se obter tumores com volume diferente ${ }^{15}$. Ao final do experimento, os dados foram obtidos de diferentes estágios tumorais e a relação entre massa e volume foi definida na equação linear $(R 2=0,9892)$. Uma vez definida, a medida do peso do tumor, esses valores foram subtraídos da medida do peso corporal de cada animal por dia. Dessa forma, após realização do experimento de diagnóstico da caquexia, foi estabelecido o décimo dia para o diagnóstico de caquexia após a indução tumoral para todos os animais, assim, o grupo experimental iniciou a intervenção da AF, a cada sessão eles tinham 48 horas de intervalo para a próxima sessão. Após 48 horas da sétima sessão os animais sobreviventes foram eutanasiados.

\section{Instrumentos e procedimentos para avaliação da composição corporal}

Após 26 dias do início do experimento, todos os 26 animais do grupo controle e experimental foram anestesiados com cetamina/xilazina $(75 \mathrm{mg} / \mathrm{kg}$ e $5 \mathrm{mg} / \mathrm{kg}$ peso, respectivamente) e eutanasiados por luxação cervical ${ }^{16}$, para realizar a coleta de tecidos musculares esqueléticos do quadríceps femoral. Os tecidos coletados foram pesados usando uma balança digital de precisão analítica (A. Cientifica EEQ9003E) ${ }^{17}$.

\section{Instrumento e procedimentos da análise histoló- gica do músculo quadríceps femoral}

Amostras biológicas colhidas (músculo quadríceps femoral) foram pesados individualmente, seccionados e colocados parte em solução fixadora de paraformaldeído $4 \%$ (p:v), tempo de fixação de $4 \mathrm{~h}$, à temperatura ambiente e parte em RNA holder e tissue tek para congelamento a $-80{ }^{\circ} \mathrm{C}$. As amostras fixadas em solução de formalina tamponada a $4 \%$ foram emblocadas em parafina e foram submetidas a cortes microscópicos para a realização dos estudos morfométricos.

Tecidos musculares esqueléticos fixados em formol e implantados em parafina dos membros traseiros direito (quadríceps femoral) foram submetidos à cortes de $7 \mu \mathrm{m}$ e marcado em Hematoxilina-Eosina (HE). Seções dos músculos esqueléticos foram feitas a 90 o e 180 o para o eixo longitudinal das fibras musculares. Pelo menos três distintos indicados, aleatoriamente, campos microscópicos dos tecidos musculares esqueléticos por animal foram fotografados. Secções do músculo foram fotografadas usando um microscópio Olympus BX50 (Olympus Corp., JAP). A área da fibra muscular transversal foi medida usando o software Image J (imagej.nih.gov). Todos os dados são expressos como a média \pm SEM. Foram analisadas e fotografadas 3 lâminas do tecido muscular de cada animal, na mesma lâmina, foram fotografados 5 locais distintos.

\section{Análise Estatística}

Todos os dados coletados foram analisados estatisticamente no software SPSS (Statistical Package for the Social Sciences) versão 20.0. O nível de confiança adotado em todas as análises foi fixado em $95 \%$ 
$(p<0.05)$. Foi realizado o teste de Shapiro-Wilk para verificação da normalidade, posteriormente, foi selecionado o teste T de Student independente para análise inferencial das variáveis dependentes.

\section{Resultados}

Os resultados da Fig. 1 mostram a análise histomorfométrica da área da fibra do músculo quadríceps femoral do membro traseiro direito de camundongos C57BL/6 do modelo tumoral singênico murino de melanoma cutâneo, a análise inferencial utilizando o teste T de Student independente não mostra diferença significativa $(p<0,05)$ entre os grupos, mas a área média da fibra muscular do CRC-ATF foi maior que do grupo controle.

Figura 1. Análise da área da fibra do músculo quadríceps femoral do membro traseiro direito de camundongos C57BL/6 do modelo tumoral singênico murino de melanoma cutâneo e com ocorrência de caquexia submetidos à atividade física em ambiente enriquecido e controles
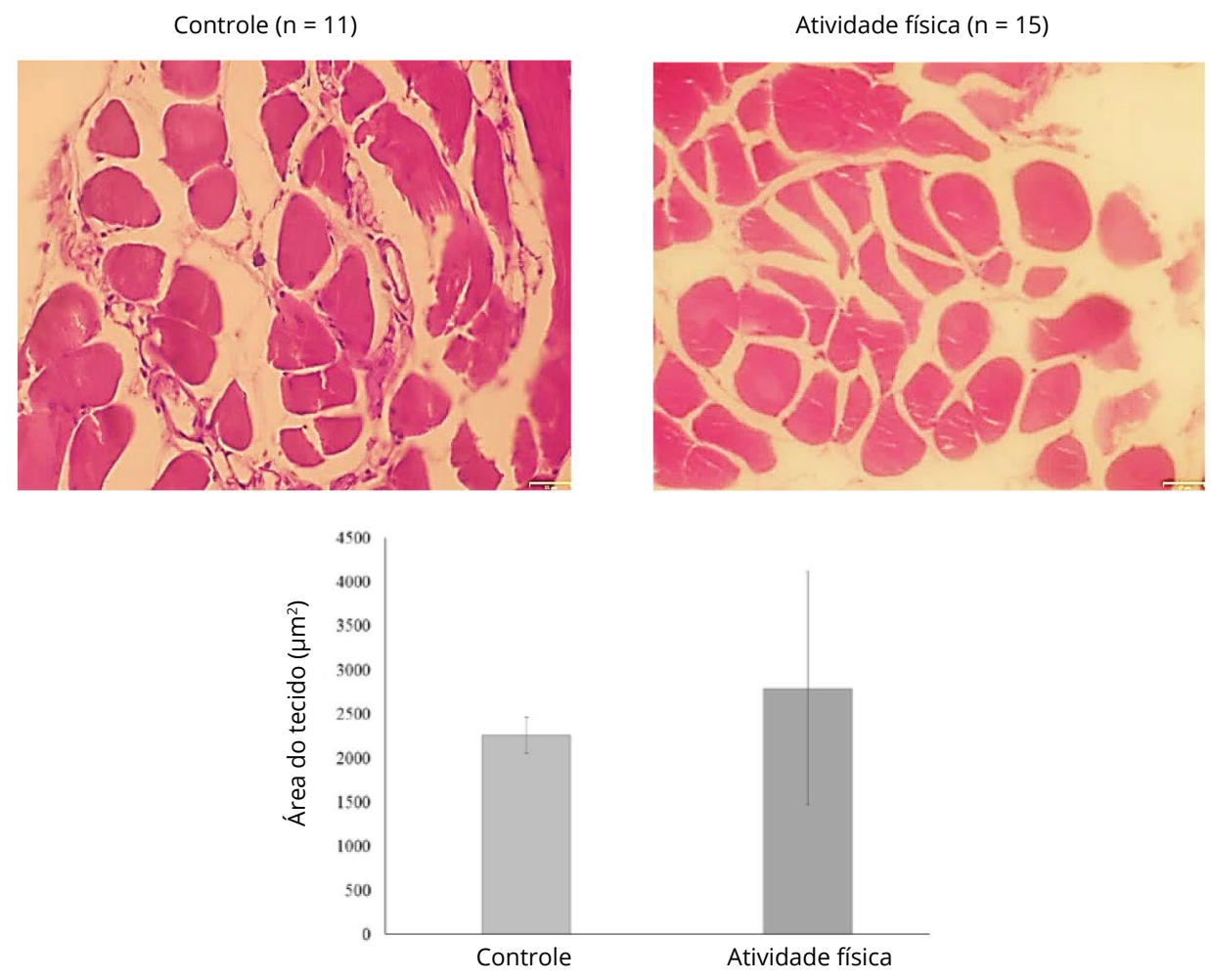

\section{Discussão}

Em estudo realizado por Rodrigues et al. ${ }^{\frac{13}{3}}$ utilizando diversos modelos, dentre eles o modelo de atividade física em ambiente enriquecido com camundongos fêmeas C57BL/6 saudáveis, os resultados mostraram que ocorreram contribuições na força muscular, composição corporal, e na histomorfometria. Porém, essa diferença não foi significativa comparado com o grupo controle.

O estado de atrofia muscular na caquexia associada ao câncer ocorre pela intensa atividade de catabolismo no corpo do "hospedeiro" do tumor provocando uma resposta inflamatória causada pelo aumento das citocinas (TNF-a e IL-6). Essa inflamação estabelece uma nova priorização no metabolismo de macronutrientes, promovendo o aumento do estresse oxidativo e do sistema proteolítico ubiquitina-proteassoma, ambas responsáveis pela degradação de proteínas, causando assim a diminuição da síntese proteica, utilizada como fonte para atender às demandas metabólicas aumentadas no hospedeiro causada pela presença do tumor $5,18,6,3$. 
O tamanho médio da fibra muscular dos animais do grupo inervação foi maior que no grupo sem intervenção, apesar da não diferença significativa, os resultados são expressivos, os resultados mostram que provavelmente é possível preservar a estrutura muscular, pois os melhores resultados foram encontrados com os animais que participaram da atividade física em ambiente enriquecido, provavelmente em função de reduzir a ação do TNFa e IL-6 e aumentar a atividade das enzimas superóxido de mustase (SOD), glutationa perioxidase (GPx) e Mn-SOD mitocondrial, causando uma ação protetora contra o dano celular de espécies reativa de oxigênio (ROS) e aumentando a taxa de síntese proteica ${ }^{19}$. Corroborando com Ballarò et al. ${ }^{20}$, o exercício moderado é capaz de amenizar a diminuição da massa muscular e evitar a perda de força muscular, pois tal situação foi associada a redução níveis de espécies reativas de oxigênio, nesse trabalho foi investigado o estresse oxidativo na presença e ausência de treinamento aeróbio moderado em camundongos caquéticos albinos Balb/c com carcinoma colorretal.

Achados de necropsia de seres humanos, em vários músculos revelaram, que após casos de caquexia, as fibras apresentavam atrofia predominantemente II com fibras angulares ocasionais; apresentando-se em grupos extensos, maior em membros inferiores ${ }^{21}$.

Apesar dos achados serem interessantes, a ausência grupo controle e o tamanho amostral pequeno são limitadores para outras indagações, porém, é de grande importância cientifica os resultados aqui apresentados. Torna-se necessário propor novas pesquisas para elucidar as lacunas aqui deixadas.

\section{Conclusões}

Os resultados deste presente estudo, mostram que não houve alterações significativas na área da fibra do músculo quadríceps femoral, em camundongos caquéticos, que realizaram atividade física em ambiente enriquecido, quando comparados com o grupo controle.

\section{Agradecimentos}

À Fundação de Amparo à Pesquisa do Estado de Minas Gerais (FAPEMIG); Conselho Nacional de Desenvolvimento Científico e Tecnológico (CNPq) e a Coordenação de Aperfeiçoamento de Pessoal de Nível Superior (CAPES) pelo apoio à realização deste estudo.

\section{Contribuições dos autores}

Santos KL, Lopes JVN e Sousa BVO realizaram os experimentos no biotério. Oliveira TR delineou o método estatístico necessário ao estudo. Freitas AS e Vieira MM realizaram as histologias do tecido muscular. Alves MR e Rodrigues VD orientaram e coordenaram o trabalho.

\section{Conflitos de interesses}

Nenhum conflito financeiro, legal ou político envolvendo terceiros (governo, empresas e fundações privadas, etc.) foi declarado para nenhum aspecto do trabalho submetido (incluindo, mas não se limitando a subvenções e financiamentos, participação em conselho consultivo, desenho de estudo, preparação de manuscrito, análise estatística, etc.).

\section{Referências}

1. Wainstein AJA, Belfort FA. Conduta para o melanoma cutâneo. Rev Col Bras Cir. 2004;31(3):204-214. doi: 10.1590/S0100$\underline{69912004000300011}$

2. Matheus LGM, Verri BHMA. Aspectos epidemiológicos do melanoma cutâneo. Revista Ciência e Estudos Acadêmicos de Medicina. 2015;(03):10-24.

3. Fearon $\mathrm{KCH}$. Cancer cachexia: developing multimodal therapy for a multidimensional problem. Eur J Cancer. 2008;44(8):1124-32. doi: 10.1016/j.ejca.2008.02.033

4. Argilés JM, Busquets S, Stemmler B, López-Soriano FJ. Cancer cachexia: understanding the molecular basis. Nat Rev Cancer. 2014;14(11):754-62. doi: 10.1038/nrc3829

5. Tisdale MJ. Mechanisms of cancer cachexia. Physiol Rev. 2009;89(2):381-410. doi: 10.1152/physrev.00016.2008

6. Salomão EM. Atividade Física Associada ao Crescimento Tumoral e Suplementação Nutricional: Estudo em ratos jovens portadores do Carcinossoma de Walker [dissertação]. São Paulo: Universidade de Campinas-Unicamp; 2005.

7. Falconer JS, Fearon KC, Plester CE, Ross JA, Carter DC. Cytokines, the acute-phase response, and resting energy expenditure in cachectic patients with pancreatic cancer. Ann Surg. 1994;219(4):325-331. doi: 10.1097/00000658-199404000-00001 
8. Bacurau RFP, Rosa LFBPC. Efeitos do exercício sobre a incidência e desenvolvimento do câncer. Rev Paul Educ Fís. 1997;11(2):142-47.

9. Al-Majid S, McCarthy DO. Cancer-induced fatigue and skeletal muscle wasting: the role of exercise. Biol Res Nurs. 2001;2(3):18697. doi: $10.1177 / 109980040100200304$

10. Plata-Salamán CR. Anorexia during acute and chronic disease. Nutrition. 1996;12(2):69-78. doi: 10.1016/s0899-9007(96)90702-9

11. Aguiar Jr AS, Pinho RA. Efeitos do exercício físico sobre o estado redox cerebral. Revista Brasileira de Medicina do Esporte. 2007;13(5):355-360. doi: 10.1590/S1517-86922007000500014

12. Rodrigues VD. Efeitos do treinamento resistido e da atividade física em camundongos C57BL/6 com caquexia associada ao modelo tumoral singênico de melanoma cutâneo [tese]. Minas Gerais: Universidade Estadual de Montes Claros; 2018.

13. Rodrigues VD, Pimentel DM, Brito AS, Vieira MM, Santos AR, Machado AS et al. Methodological validation of a vertical ladder with low intensity shock stimulus for resistance training in C57BL/6 mice: Effects on muscle mass and strength, body composition, and lactate plasma levels. J Hum Sport Exerc. 2019;14(3):608-631. doi: 10.14198/jhse.2019.143.12

14. Voltarelli FA, Frajacomo FT, Padilha CS, Testa MTJ, Cella PS, Ribeiro DF et al. Syngeneic B16F10 melanoma causes cachexia and impaired skeletal muscle strength and locomotor activity in mice. Frontiers in physiology. 2017;8:715. doi: 10.3389/ fphys.2017.00715
15. Honors MA, Kinzig KP. Characterization of the Yoshida sarcoma: a model of cancer cachexia. Support Care Cancer. 2013;21(10):2687-2694. doi: 10.1007/s00520-013-1839-y

16. Murton AJ, Constantin D, Greenhaff PL. The involvement of the ubiquitin proteasome system in human skeletal muscle remodelling and atrophy. Biochim Biophys Acta. 2008;1782(12):730-43. doi: 10.1016/j.bbadis.2008.10.011

17. Groll M, Bajorek M, Köhler A, Moroder L, Rubin DM, Huber R et al. A gated channel into the proteasome core particle. Nature structural biology. 2000;7(11):1062-7. doi: 10.1038/80992

18. Barreiro E, Puente BL, Busquets S, López-Soriano FJ, Gea J, Argilés JM. Both oxidative and nitrosative stress are associated with muscle wasting in tumour-bearing rats. FEBS Lett. 2005;579(7):1646-52. doi: 10.1016/j.febslet.2005.02.017

19. Gould DW, Lahart I, Carmichael AR, Koutedakis Y, Metsios GS. Cancer cachexia prevention via physical exercise: molecular mechanisms. J Cachexia Sarcopenia Muscle. 2013;4(2):111-124. doi: $10.1007 / \mathrm{s} 13539-012-0096-0$

20. Ballarò R, Penna F, Pin F, Gómez-Cabrera MC, Viña J, Costelli $P$. Moderate exercise improves experimental cancer cachexia by modulating the redox homeostasis. Cancers. 2019;11(3):285. doi: 10.3390/cancers11030285

21. Kouyoumdjian JA. Neuromuscular abnormalities in disuse, ageing and cachexia. Arq Neuro-Psiquiatr. 1993;51(3):299-306. doi: 10.1590/S0004-282X1993000300001 